NISTIR 8153

\title{
Report on the May 2016 ASTM E57.02 Instrument Runoff at NIST, Part 2 - NIST Realization of Test Procedures and Uncertainties in the Reference Lengths
}

\author{
Bala Muralikrishnan \\ Prem Rachakonda \\ Meghan Shilling \\ Vincent Lee \\ Christopher Blackburn \\ Daniel Sawyer \\ Geraldine Cheok \\ Luc Cournoyer
}




\section{Report on the May 2016 ASTM E57.02 Instrument Runoff at NIST, Part 2 - NIST Realization of Test Procedures and Uncertainties in the Reference Lengths}

Bala Muralikrishnan, Prem Rachakonda, Meghan Shilling, Vincent Lee, Christopher Blackburn, Daniel Sawyer Engineering Physics Division Physical Measurement Laboratory NIST

Geraldine Cheok Intelligent Systems Division Engineering Laboratory NIST

Luc Cournoyer Measurement Science and Standards, National Research Council of Canada, Ottawa, Canada

This publication is available free of charge from: https://doi.org/10.6028/NIST.IR.8153

October 2016

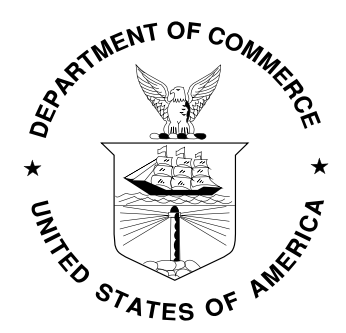

U.S. Department of Commerce

Penny Pritzker, Secretary 


\section{Disclaimer}

Commercial equipment and materials may be identified in order to adequately specify certain procedures. In no case does such identification imply recommendation or endorsement by the National Institute of Standards and Technology, nor does it imply that the materials or equipment identified are necessarily the best available for the purpose. 


\section{Table of contents}

1. NIST realization of test procedures

2. Targets

2.1. Targets used for the grid and inside test setup

2.2. Targets used for the relative range tests

2.2.1. The plate-sphere artifact

2.2.2. The bare-sphere artifact

3. Grid of spheres

3.1. Test setup

3.2. Calibrating the long horizontal length $(\mathrm{DH})$

3.2.1. The four-azimuth averaging procedure

3.2.2. Validation experiments

3.2.3. Uncertainty budget

3.2.4. Providing reference measurements for the grid

3.3. Calibrating the central nine spheres of the grid

3.3.1. Calibration procedure

3.3.2. Validation experiments

3.3.3. Uncertainty budget

4. Inside test

4.1. Test setup

4.2. Calibration procedure

4.3. Validation experiments

4.4. Uncertainty budget

5. Relative range tests

5.1. Test setup

5.2. Uncertainty budget

6. Summary

7. Acknowledgements

8. References 


\section{NIST realization of the test procedures}

There is ongoing activity within ASTM E57.02 working group WK43218 [1] to develop a documentary standard for point-to-point distance performance evaluation of spherical coordinate 3D imaging systems. The Dimensional Metrology Group (DMG) at the National Institute of Standards and Technology (NIST) has established facilities to realize all of the test procedures in the current draft.

Part 1 [2] of this two-part report presented background information and key findings from an instrument runoff hosted by the DMG in support of this effort. This report (Part 2) describes test facilities established by the DMG, calibration procedures, validation data, and uncertainty budgets. It is important to note that the eventual standard will not mandate how a user will realize any of the test procedures; this report merely describes the approach adopted by the DMG at NIST. It is also noted that this report addresses the uncertainties in the reference lengths only; the overall test uncertainty may have other components that are not addressed here.

In order to realize all tests described in the draft standard (which are also given in Appendix A of Part 1 [2] of the report), DMG established three setups in two laboratories. A calibrated grid of spheres and an inside test setup are established in the large-scale laboratory, and a relative range setup is established in the tape-tunnel facility. Section 2 describes the targets used, while subsequent sections describe each of the three setups. A laser tracker is used as the reference instrument (RI) to calibrate the test setups. For readers not familiar with the laser tracker technology, a review can be found in [3].

\section{Targets}

\subsection{Targets used for the grid and the inside test setup}

"Integration spheres" manufactured by Bal-tec Inc. are used in the grid and the inside test setup. The integration spheres are partial spheres made of aluminum with a satin finish that is suitable for 3D imaging systems. The reflectance factor of a sample with similar finish (also provided by the same manufacturer) is measured to be 0.27 at a wavelength of 710 $\mathrm{nm}$ and 0.36 at a wavelength of $1500 \mathrm{~nm}$. The 3D imaging systems involved in the runoff operated at a wavelength of approximately $700 \mathrm{~nm}$ or $1500 \mathrm{~nm}$.

A smaller sphere is less expensive and easier to handle but a larger sphere allows for more data to be obtained from the surface; spheres of nominal $100 \mathrm{~mm}$ diameter are chosen as a tradeoff. The insides of the integration spheres are hollowed out and kinematic mounting balls are located to centrally seat a $38.1 \mathrm{~mm}(1.5 \mathrm{in})$ spherically mounted retroreflector (SMR) as shown in Fig. 1 (a) and (b). The SMR is held in place by a magnet inside the integration sphere.

The integration spheres may be used in two different modes. In one mode of use, the centerto-center distance between $38.1 \mathrm{~mm}$ (1.5 in) SMR nests distributed in the work volume are 
calibrated using a laser tracker and $38.1 \mathrm{~mm}$ (1.5 in) SMRs (see Fig. 1 (c)). Subsequently, the SMRs are replaced with $38.1 \mathrm{~mm}$ (1.5 in) spheres and the integration spheres are mounted on top (see Fig. 1(d)). In this mode of user, both the laser tracker and the instrument under test (IUT), the 3D imaging system, are on the same side of the target.
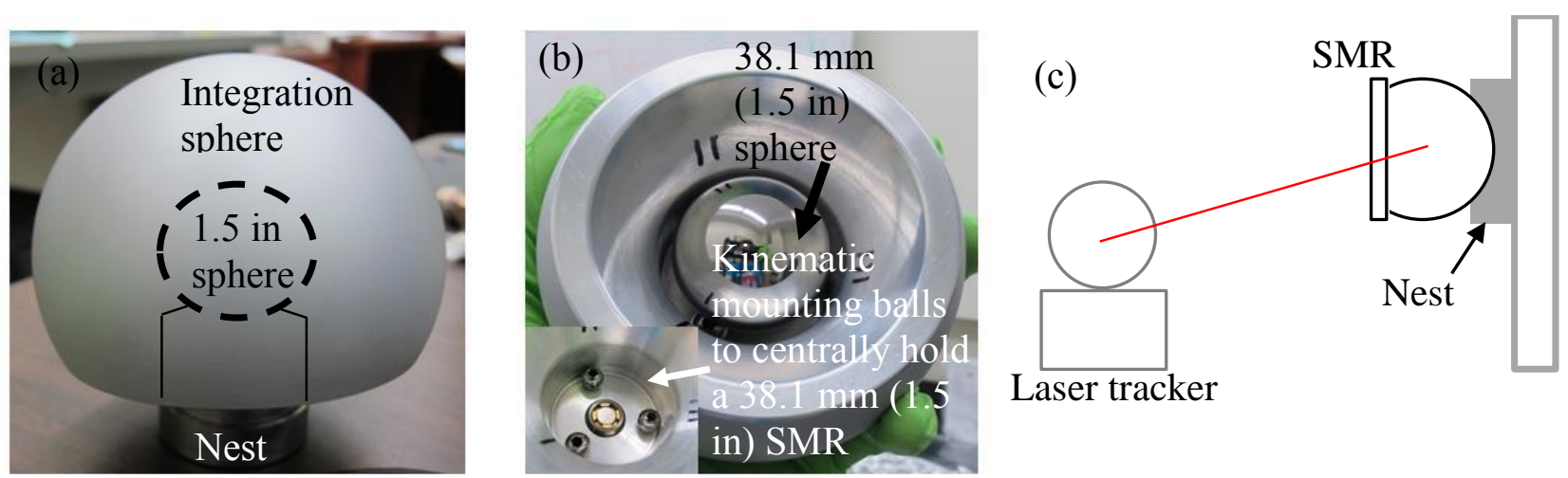

Laser tracker

(d)

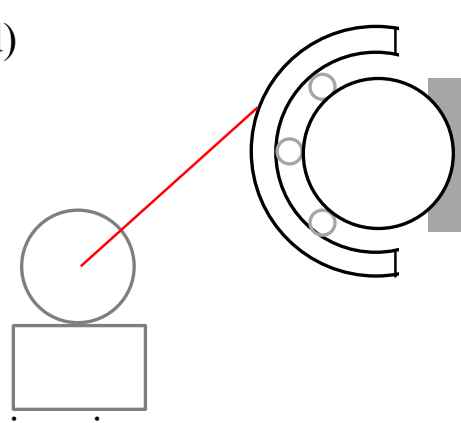

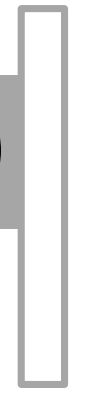

(e)

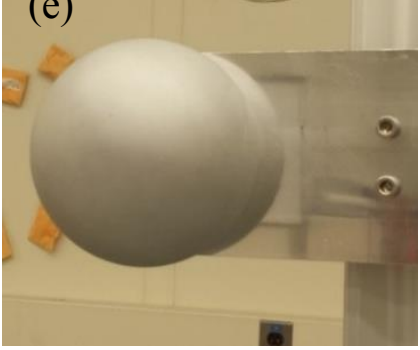

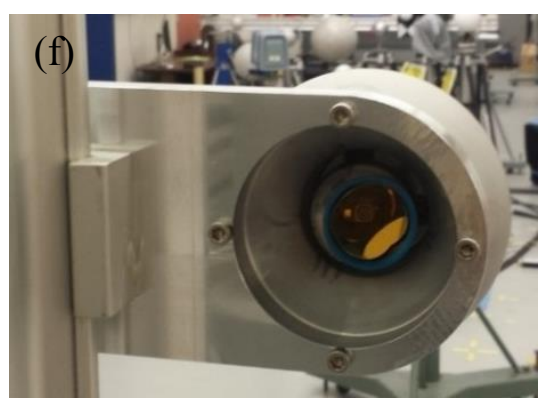

$3 \mathrm{D}$ imaging

(g)

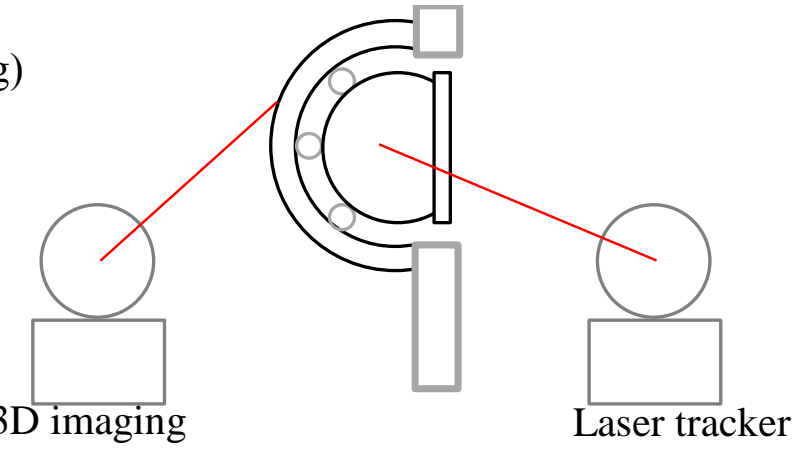

Fig. 1 (a) Integration sphere mounted on a $38.1 \mathrm{~mm}$ (1.5 in) sphere placed in the work volume, $(b)$ view from the back of the integration sphere showing the kinematic mounting balls to hold the $38.1 \mathrm{~mm}$ (1.5 in) SMR, (c) calibrating the center-to-center distance using a laser tracker and $38.1 \mathrm{~mm}$ (1.5 in) SMR, (d) the SMR is replaced with a $38.1 \mathrm{~mm}$

(1.5 in) sphere and the integration sphere is mounted on top, (e) integration sphere rigidly bolted to support plates, $(f)$ a $38.1 \mathrm{~mm}$ (1.5 in) SMR placed inside the integration sphere, $(g)$ schematic showing the calibration of the center-to-center distance from the backside while the 3D imaging system views the sphere from the front side 
In another mode of use, the integration spheres are bolted to support plates as shown in Fig. 1 (e). SMRs are placed inside the spheres as shown in Fig. 1(f). A laser tracker, placed behind the plane of the spheres, is used to calibrate the center-to-center distance while the 3D imaging system is placed in front during the performance tests (see Fig. 1(g)). This approach is adopted in the DMG laboratories primarily because the magnet inside the integration sphere sometimes does not exert a sufficiently strong force to hold the integration sphere when the sphere is mounted facing downwards, i.e., mounted in a manner so that the gravitational and the magnetic force counter each other.

For each of the 16 integration spheres procured by the DMG, a contact probe coordinate measuring machine $(\mathrm{CMM})$ is used to measure the diameter, concentricity between the outer surface and SMR center, and form of the outer surface. The results are tabulated in Table 1 . The average concentricity and form are both approximately $0.01 \mathrm{~mm}$.

Table 1 Diameter, concentricity, and form data for 16 integration spheres

\begin{tabular}{rrr}
\hline Diameter & Concentricity & Form \\
\hline$(\mathrm{mm})$ & $(\mathrm{mm})$ & $(\mathrm{mm})$ \\
\hline 100.050 & 0.009 & 0.007 \\
100.041 & 0.004 & 0.007 \\
100.017 & 0.014 & 0.010 \\
99.991 & 0.020 & 0.015 \\
99.979 & 0.012 & 0.006 \\
100.017 & 0.009 & 0.007 \\
100.018 & 0.004 & 0.006 \\
100.003 & 0.012 & 0.009 \\
99.998 & 0.011 & 0.010 \\
100.004 & 0.016 & 0.007 \\
100.013 & 0.007 & 0.008 \\
99.988 & 0.019 & 0.017 \\
99.991 & 0.010 & 0.011 \\
99.990 & 0.011 & 0.010 \\
100.006 & 0.001 & 0.007 \\
99.983 & 0.005 & 0.011 \\
\hline
\end{tabular}

\subsection{Targets used for the relative range tests}

The relative range tests are performed using two different targets, a plate-sphere artifact and a bare-sphere artifact.

\subsubsection{The plate-sphere artifact}

The plate-sphere artifact consists of a $609.6 \mathrm{~mm} \times 609.6 \mathrm{~mm}(2 \mathrm{ft} \times 2 \mathrm{ft})$ plate with a nominal $200 \mathrm{~mm}$ diameter integration sphere mounted in the middle. Plate material from a 
$50.8 \mathrm{~mm}$ ( $2 \mathrm{in}$ ) annular region around the sphere is removed to reduce the probability of secondary reflections from influencing the 3D imaging system's range measurement.

The purpose of the sphere on the plate is only to serve as a fiducial, i.e., to tie the platecenter as determined by the 3D imaging system to the corresponding point measured by the laser tracker, which is the center of the SMR centrally located inside the sphere. This registration is achieved as follows:

- Both the plate and the sphere are scanned using the 3D imaging system and the data representing the two geometries are separated

- A least-squares plane is fit to the data corresponding to the plate and the normal vector determined

- A least-squares sphere is fit to the data corresponding to the sphere and the sphere center is obtained

- The sphere center is then projected on to the plate along the normal vector of the plane to determine the plate-center.

Because the artifact is designed so that the mechanical center of the sphere lies on the front surface of the plate, the plate-center as determined above is theoretically coincident with the center of the SMR. Ensuring coincidence of the plate-center and the SMR minimizes the Abbe error that can occur if the plate is misaligned (i.e., not orthogonal to the line joining the laser tracker and the 3D imaging system).

Fig. 2 shows a schematic and three different views of the plate-sphere artifact used during the runoff. The flatness of the plate is measured on a CMM and determined to be $60 \mu \mathrm{m}$. The diameter, concentricity, and form error of the sphere are determined by CMM measurements to be $199.996 \mathrm{~mm}, 0.003 \mathrm{~mm}$, and $0.011 \mathrm{~mm}$ respectively. The center of the sphere is $0.126 \mathrm{~mm}$ below the surface of the plate.
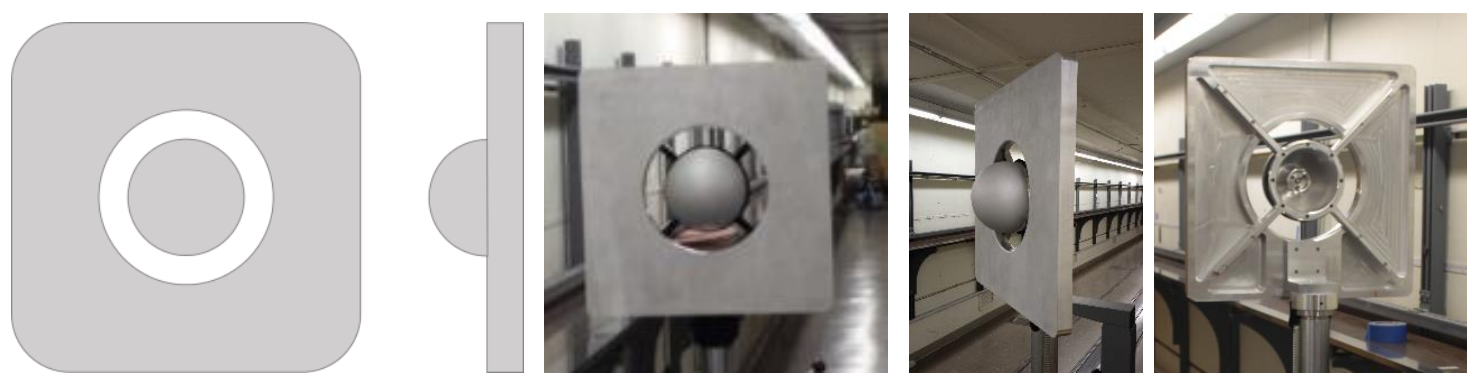

Fig. 2 Different views of the plate-sphere artifact used during the runoff

\subsubsection{The bare-sphere artifact}

The bare sphere artifact consists of a nominal $200 \mathrm{~mm}$ diameter integration sphere mounted on a plate using $101.6 \mathrm{~mm}$ (4 in) standoffs as shown in Fig. 3. The purpose of the standoffs is to reduce the probability of secondary reflections from the background surface from influencing the 3D imaging system's range measurement. The support plate is covered with laser absorbing black felt material to further reduce the potential for secondary scattering. 
The diameter, concentricity, and form error of the sphere are determined by CMM measurement to be $200.003 \mathrm{~mm}, 0.006 \mathrm{~mm}$, and $0.004 \mathrm{~mm}$, respectively.

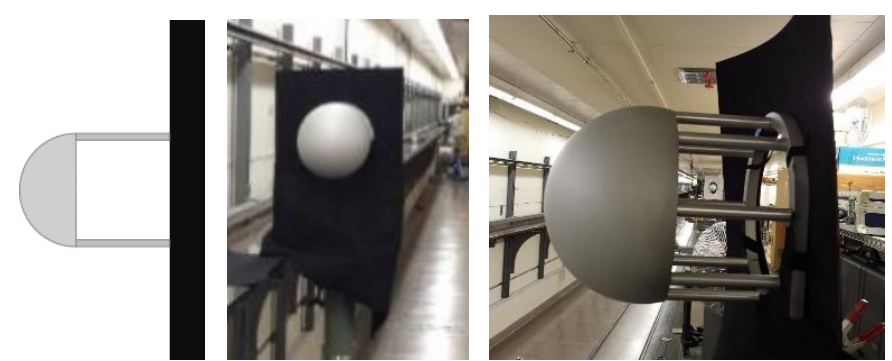

Fig. 3 Bare-sphere artifact used during the runoff.

\section{Grid of spheres}

\subsection{Test setup}

Eleven integration spheres are arranged in the large-scale laboratory as shown in Fig. 4 to realize all of the two-face, the near symmetric, the near asymmetric, and far symmetric point-to-point length tests (see Appendix A of Part 1 [2] for a list of the tests). Each of the three vertical rails attached to tall stands hold three integration spheres. The two integration spheres on the extremes (D and H) are mounted directly on shorter stands. The horizontal and vertical spacing between spheres is about $1.6 \mathrm{~m}$. The 3D imaging system is placed in front of the grid during the tests. Eleven SMRs are placed, one inside each integration sphere, to allow for the calibration of the center-to-center distance using a laser tracker in absolute distance measurement (ADM) mode.

While a single laser tracker placed behind the grid may be used to calibrate the entire grid of 11 spheres, a two-step procedure is adopted to reduce the uncertainty in the sphere center-to-center distance calibration. In the first step, the two extreme spheres are calibrated using a laser tracker through a method described in the next section. In the second step, the central nine spheres of the grid are calibrated from multiple positions of a laser tracker as described in Section 3.3. 


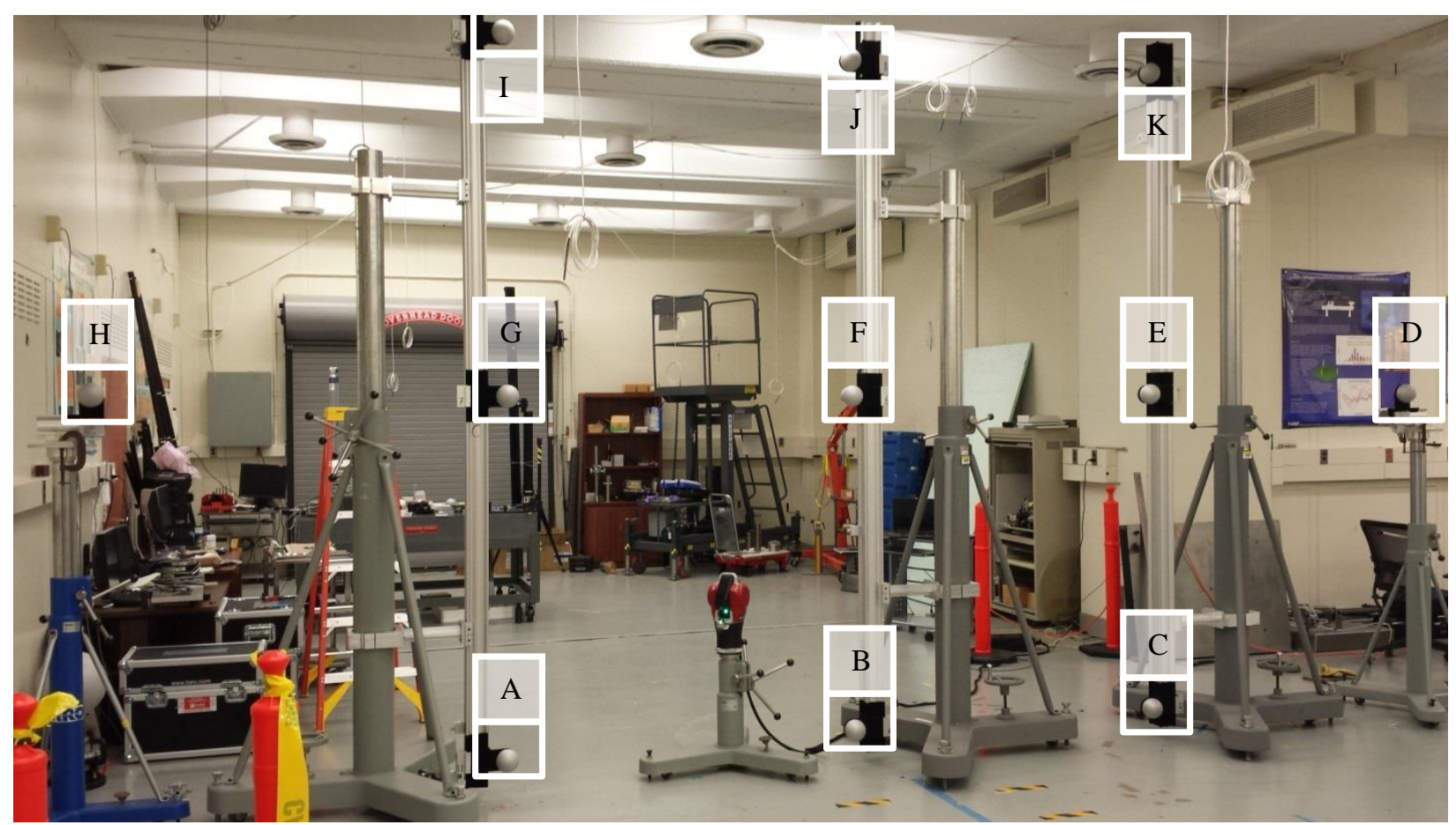

Fig. 4 A grid of 11 spheres in the large-scale laboratory at NIST

\subsection{Calibrating the long horizontal length (DH)}

\subsubsection{The four-azimuth averaging procedure}

A tracker is placed centrally behind the grid, as close as possible to the grid (which happens to be about $5 \mathrm{~m}$ to avoid clipping the beam at the edges of the integration spheres), and at approximately the same height as the central horizontal row of integration spheres as shown in Fig. 5(a). All measurements are performed using the ADM mode of the tracker. Frontface and back-face measurements are averaged to determine coordinates of centers of integration spheres $\mathrm{D}$ and $\mathrm{H}$, and the length between $\mathrm{D}$ and $\mathrm{H}$ is determined. The tracker is then rotated about its vertical axis by $90^{\circ}$ and the length is determined again. This process is repeated two more times for a total of four lengths, one collected at each of the four azimuth angles $0^{\circ}, 90^{\circ}, 180^{\circ}$, and $270^{\circ}$. The average of the four measured lengths is within few micrometers from an interferometric (IFM) line-of-sight measurement, as the validation data and uncertainty budget below shows.

This procedure is based on work reported in [3]. Averaging front- and back-face measurements removes errors associated with terms that reverse in sign between front-face and back-face. Any remaining tracker error model parameters are trigonometric functions and therefore periodic; averaging measurements from four azimuth positions $90^{\circ}$ apart removes the influence of these terms. Ranging errors will also manifest in the measurements, and this is accounted for in the uncertainty budget discussed in Section 3.2.3. 

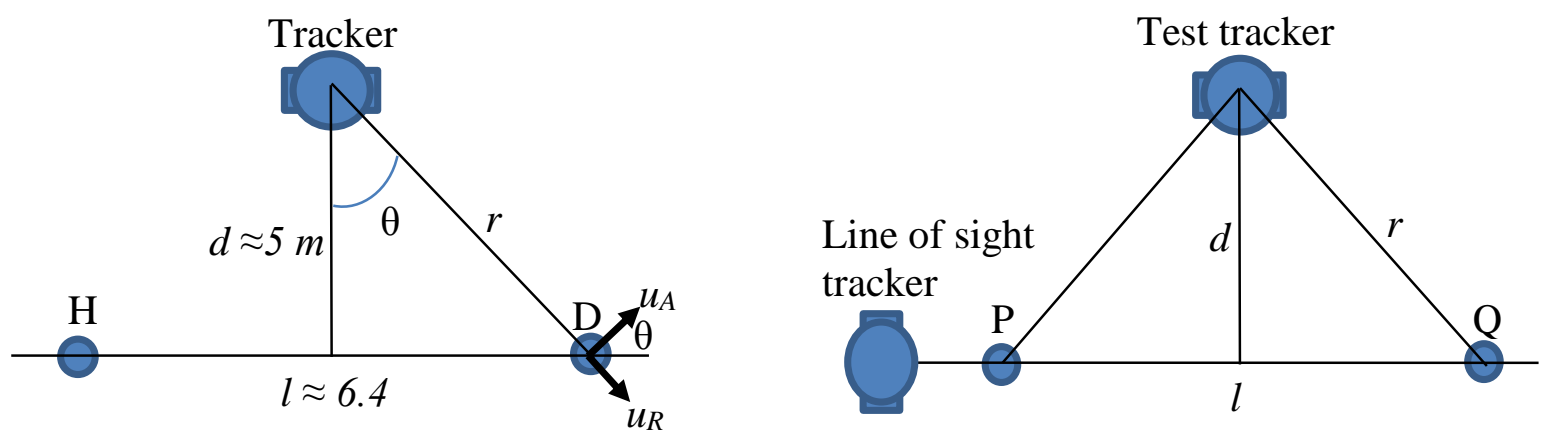

Fig. 5 (a) Setup to measure a horizontal length (top view shown) between integration spheres $D$ and $H,(b)$ establishing the validity of the method using a test setup

\subsubsection{Validation experiments}

In order to establish the validity of the four-azimuth averaging procedure, two $38.1 \mathrm{~mm}$ (1.5 in) nests $\mathrm{P}$ and $\mathrm{Q}$ are mounted on stands a distance $l$ apart with the test tracker centrally placed at a distance $d$ from the line joining $\mathrm{P}$ and Q as shown in Fig. 5(b). Table 2 shows the different values of $l$ and $d$ used for the validation. The distance between the nests is calibrated using a line-of-sight tracker (in IFM mode) placed along the line joining the two nests. The standard uncertainty in line-of-sight calibrations with laser trackers is on the order of $10 \mu \mathrm{m}$ without considering thermal effects. Because the horizontal and vertical axes do not need to move in line-of-sight measurements, the dominant term is the uncertainty in the range measurement of the tracker.

Table 2 Validation data for four-azimuth averaging procedure

\begin{tabular}{|l|l|l|l|l|l|}
\hline & $\begin{array}{l}l \\
(\mathrm{~m})\end{array}$ & $\begin{array}{l}d \\
(\mathrm{~m})\end{array}$ & $\begin{array}{l}\text { Line-of-sight } \\
\text { reference } \\
(\mathrm{mm})\end{array}$ & $\begin{array}{l}\text { Four-azimuth } \\
\text { averaging } \\
(\mathrm{mm})\end{array}$ & $\begin{array}{l}\text { Error } \\
(\mathrm{mm})\end{array}$ \\
\hline $\begin{array}{l}\text { Tracker A is along line- } \\
\text { of-sight, Tracker B is test } \\
\text { tracker }\end{array}$ & 7.2 & 6 & 7178.183 & 7178.177 & -0.006 \\
\cline { 2 - 6 } $\begin{array}{l}\text { Tracker B is along line- } \\
\text { of-sight, Tracker A is test } \\
\text { tracker }\end{array}$ & 7.2 & 5 & 7178.183 & 7178.175 & -0.008 \\
\cline { 2 - 6 } $\begin{array}{l}\text { Tracker A is along line- } \\
\text { of-sight, Tracker C is test } \\
\text { tracker }\end{array}$ & 7.2 & 5 & 7178.269 & 7178.265 & -0.004 \\
\cline { 2 - 6 } & 2.3 & 5 & 2328.967 & 2328.963 & -0.004 \\
\hline
\end{tabular}

The line-of-sight measurement result, the distance obtained from the four-azimuth averaging procedure (using the test tracker), and the difference between the two measurements are shown for different values of $l$ and $d$ in Table 2. Combinations of three different trackers are used for this testing. The differences are all smaller than $10 \mu \mathrm{m}$, indicating that the four-azimuth averaging procedure is capable of calibrating a horizontal length with uncertainties that are comparable to a line-of-sight calibration. The observed differences in the two methods are well below the standard uncertainty (tracker 
contribution and other mechanical terms) of $18 \mu \mathrm{m}$ associated with these measurements in the uncertainty budget of Section 3.2.3, giving confidence that the standard uncertainty used for the tracker contribution is adequate.

\subsubsection{Uncertainty budget}

Mechanical terms: The uncertainty in locating a single point in space resulting from error sources associated with the laser tracker, the centering of the integration sphere with the SMR, and the concentricity of optical and mechanical center of the SMR is first determined. The point coordinate uncertainty is then propagated to estimate the uncertainty in the point-to-point length. Uncertainty calculations are shown here for the following dimensions: $l=6.4 \mathrm{~m}$ and $d=5 \mathrm{~m}$, therefore $r=5.9 \mathrm{~m}$ and $\theta=32.6^{\circ}$ (see Fig. 5(a)).

Tracker contribution: The uncertainty along the ranging direction can be obtained from the manufacturer's specifications. For an ADM tracker with a specification of $16 \mu \mathrm{m}+0.8 \mu \mathrm{m} / \mathrm{m}$, the maximum permissible error (MPE) at a range of $5.9 \mathrm{~m}$ is $20.7 \mu \mathrm{m}$. The R0 specification for the same tracker is $16 \mu \mathrm{m}$. Summing in quadrature and assuming these form the upper bounds of a rectangular distribution, the standard uncertainty $u_{R}$ along the ranging direction is $15.1 \mu \mathrm{m}$. In order to compute the uncertainty along the direction transverse to the laser beam, only the repeatability of the angular axis is considered, not the overall accuracy of the axis. This is because all targets are measured in front- and back-face and averaged, and the length itself is the average from four azimuth orientations of the tracker separated by $90^{\circ}$ each. This removes the influence of all non-ranging systematic sources of error in the tracker. Experimentally determined one standard deviation repeatability of the horizontal angle measurement is $0.1 \mathrm{mdeg}$ (based on 10 consecutive tracker measurements of target $\mathrm{H}$ ). The vertical angle repeatability does not affect this measurement because the reference length is oriented horizontally. This translates to a one standard uncertainty $u_{A}$ of $10.4 \mu \mathrm{m}$. The standard uncertainty in the determination of coordinate $\mathrm{H}$ along the direction of the length is $\sqrt{\left(u_{R} \sin \theta\right)^{2}+\left(u_{A} \cos \theta\right)^{2}}$, or $12 \mu \mathrm{m}$.

Integration sphere concentricity with SMR: The concentricity of all 14 integration spheres are measured on a CMM. The experimentally measured one standard deviation is $5 \mu \mathrm{m}$ and this value is considered as the standard uncertainty in the determination of the coordinate.

SMR concentricity: The concentricity specification of the maximum distance between physical and optical center of the SMRs is $2.5 \mu \mathrm{m}$. Assuming this value as the upper bound with any value within that bound as equally probable (rectangular distribution), the standard uncertainty in the measured coordinate is $1.4 \mu \mathrm{m}$.

Standard uncertainty in the length: The combined standard uncertainty in the determination of a coordinate is therefore the root sum square of the three terms above, which is $13 \mu \mathrm{m}$. Because two measurements are combined to determine a length, the standard uncertainty in the length $u_{L}$ is $13 \sqrt{2}=18 \mu \mathrm{m}$. 
Thermal effects: Assuming $\pm 0.5^{\circ}$ fluctuation of the temperature in the room as an upper bound with any value inside that bound as equally probable, and assuming thermal expansion coefficient of $12 \times 10^{-6} /{ }^{\circ} \mathrm{C}$ for concrete, the standard uncertainty of a $6.4 \mathrm{~m}$ length is $22 \mu \mathrm{m}$. The uncertainty due to changes in refractive index (and therefore measured range) is not considered because the effect is an order of magnitude smaller than the uncertainty due to thermal expansion of the floor.

Combined standard uncertainty: Combining the mechanical contribution $18 \mu \mathrm{m}$ and the thermal contribution of $22 \mu \mathrm{m}$, the combined standard uncertainty in length is $28 \mu \mathrm{m}$. The expanded uncertainty $(k=2)$ is $56 \mu \mathrm{m}$.

\subsubsection{Providing reference measurements for the grid}

In addition to measuring the extreme targets $\mathrm{D}$ and $\mathrm{H}$, the remaining three targets in the horizontal row, E, F, and G, are also measured using the same four-azimuth averaging procedure. The actual method to determine calibrated lengths EF, FG, and EG is different from this four-azimuth averaging procedure, but this procedure provides these three lengths to sufficiently low uncertainty to use as reference values to aid in validating the actual procedure used (described in 3.3).

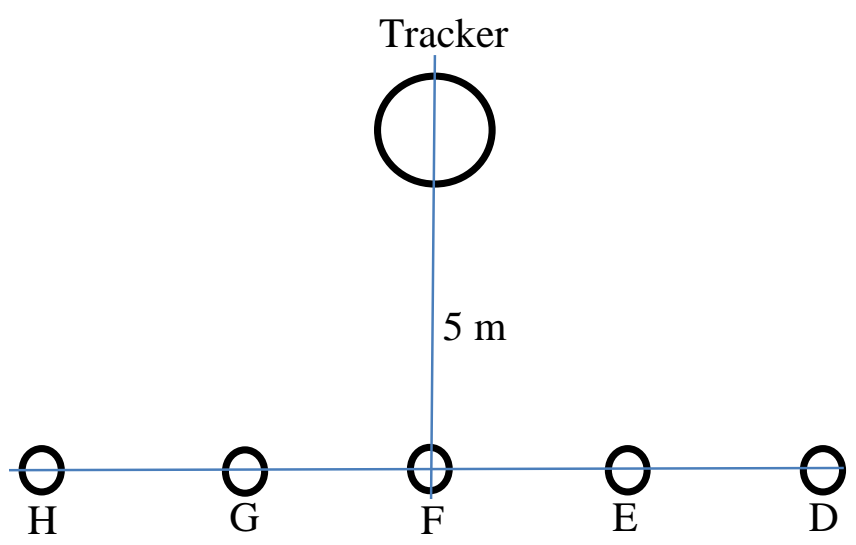

Fig. 6 The four-azimuth averaging method is used to measure coordinates of all the five targets in the central horizontal row of the grid

\subsection{Calibrating the central nine spheres of the grid}

\subsubsection{Calibration procedure}

The central nine spheres of the grid are calibrated using a laser tracker by 'averaging' the coordinate data acquired from four positions behind the grid. This averaging simply involves transforming the data from the four positions into a common frame using the method of least-squares. Sphere center-to-center distances are then calculated from the 'average' coordinates. Because it is the center-to-center distance that is the quantity of interest, it is also possible to determine these quantities for each tracker position and 
subsequently compute their average. The center-to-center distances determined using both methods yield practically the same values.

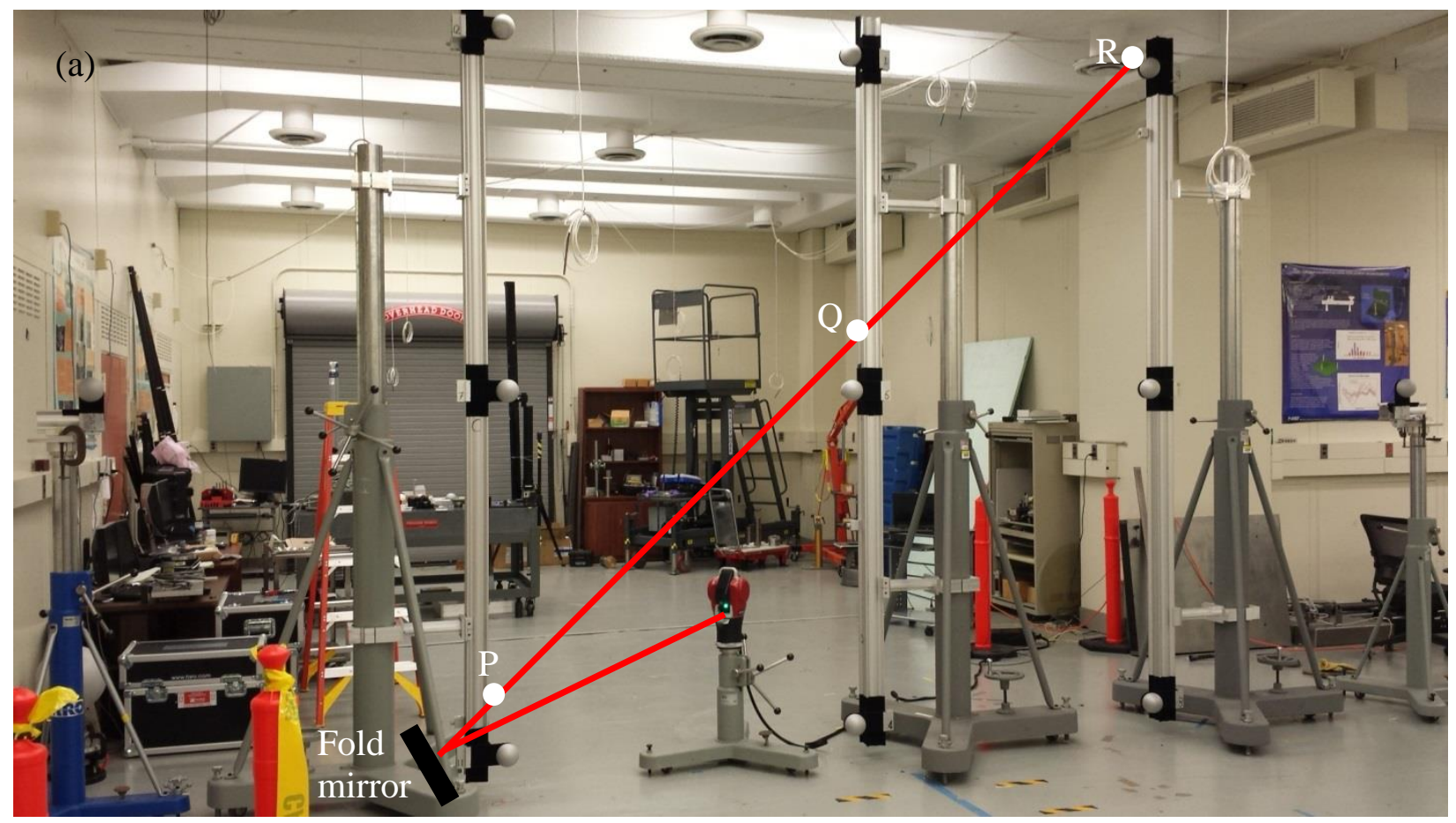

(b)
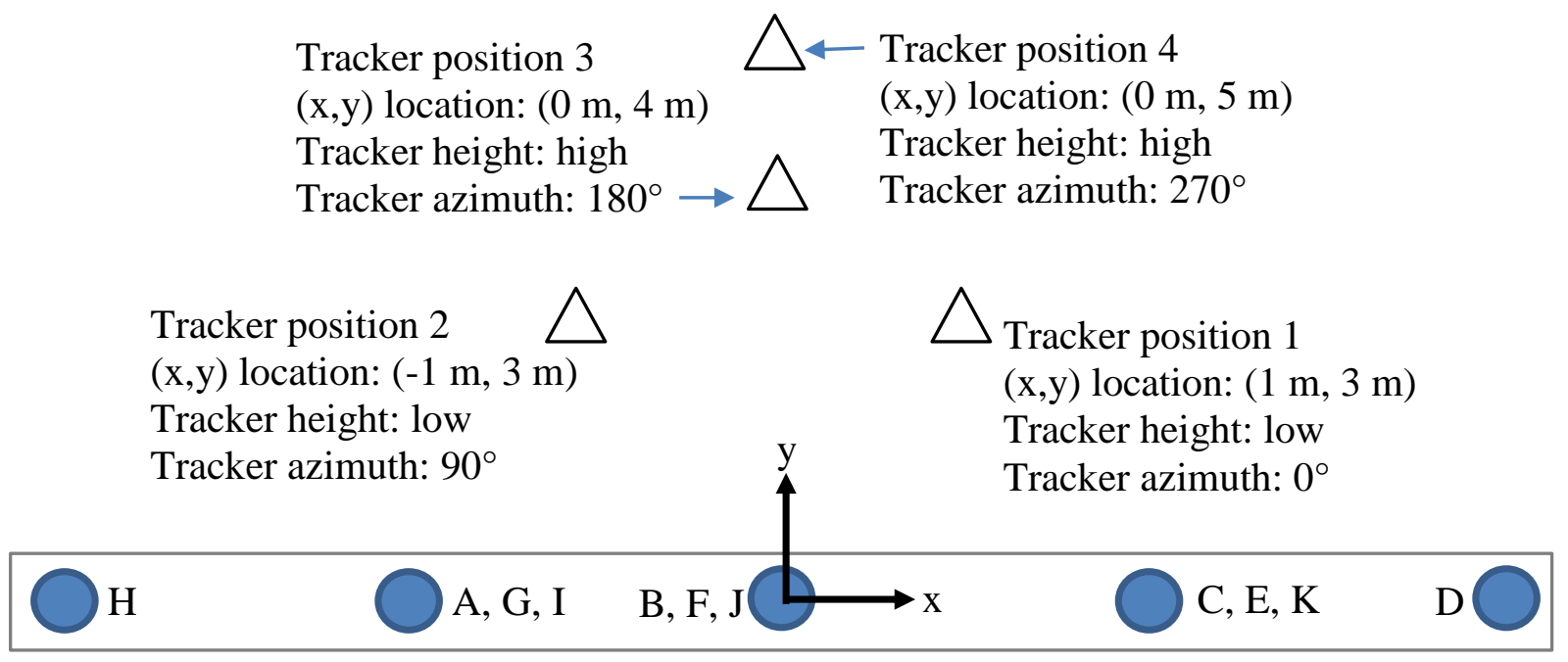

Grid of spheres (top view)

Fig. 7 (a) Three $38.1 \mathrm{~mm}$ (1.5 in) SMR nests calibrated using a line-of-sight tracker to provide additional reference lengths to validate grid calibration, (b) four nominal positions of the tracker behind the grid

In order to obtain additional reference measurements that can be used to validate the grid calibration procedure, three collinear $38.1 \mathrm{~mm}(1.5 \mathrm{in}) \mathrm{SMR}$ nests $(\mathrm{P}, \mathrm{Q}$, and $\mathrm{R})$ are set up 
behind the rails as shown in Fig. 7(a). A laser tracker is set up near nest P and aligned so that its laser beam, after bouncing off of a fold mirror on the floor, is also collinear with the three nests, allowing the tracker to be used in its most accurate line-of-sight mode. The distance between the nests is calibrated using this tracker, yielding three lengths PQ, QR, and PR.

When the nine integration spheres are measured by the laser tracker from the four positions, three additional $38.1 \mathrm{~mm}$ (1.5 in) nest locations are also measured and included as part of the averaging procedure. This grid calibration then produces coordinates of the central nine spheres and the three extra nest centers, which can be used to calculate a number of centerto-center distances. These include the horizontal lengths EF, FG, and EG, and the diagonal lengths $\mathrm{PQ}, \mathrm{QR}$, and PR. The three horizontal lengths can be compared against measurements of the same lengths obtained through the four-azimuth averaging procedure described in the previous section while the three diagonal lengths can be compared against measurements of the same lengths obtained through the line-of-sight measurement. Comparison results and long term grid stability data are presented in the next section.

To sample and average vertical angle errors in the tracker, the tracker stand is lowered for two of the positions and raised high for the remaining two positions. To sample and average horizontal angle errors in the tracker, the tracker is rotated by approximately $90^{\circ}$ about the vertical axis as it is moved from one position to the next. From each position of the tracker, all nine targets and the extra three $38.1 \mathrm{~mm}$ (1.5 in) nests are measured in front-face and back-face, and averaged to eliminate the influence of tracker model parameters that change sign from front-face to back-face. Fig. 7(b) shows the four approximate placements of the trackers behind the grid.

\subsubsection{Validation experiments}

The grid calibration is performed 14 times over a period of one month using three different trackers and three different operators. Fig. 8 shows the errors from the reference values in the three horizontal lengths EF, EG, and FG, and the three diagonal lengths PQ, PR, and QR. As mentioned earlier, the reference values for the horizontal lengths are obtained by the four-azimuth averaging method and the reference values for the diagonal lengths are obtained from the line-of-sight calibration. Reference measurements are performed immediately before every grid calibration measurement. Fig. 8 indicates that the errors are all within about $\pm 30 \mu \mathrm{m}$ over the one-month period.

In addition to the errors, the standard deviation of any given center-to-center length (based on the 14 calibrations performed over the one-month period) is computed to assess the stability of the grid. Because a total of 66 different center-to-center lengths can be computed from 12 measured coordinates (nine integration sphere centers and three 38.1 mm (1.5 in) SMR nests), the standard deviation for each of the lengths was computed. The pooled standard deviation was then determined to be $11 \mu \mathrm{m}$, indicating excellent stability of the grid over this period. 


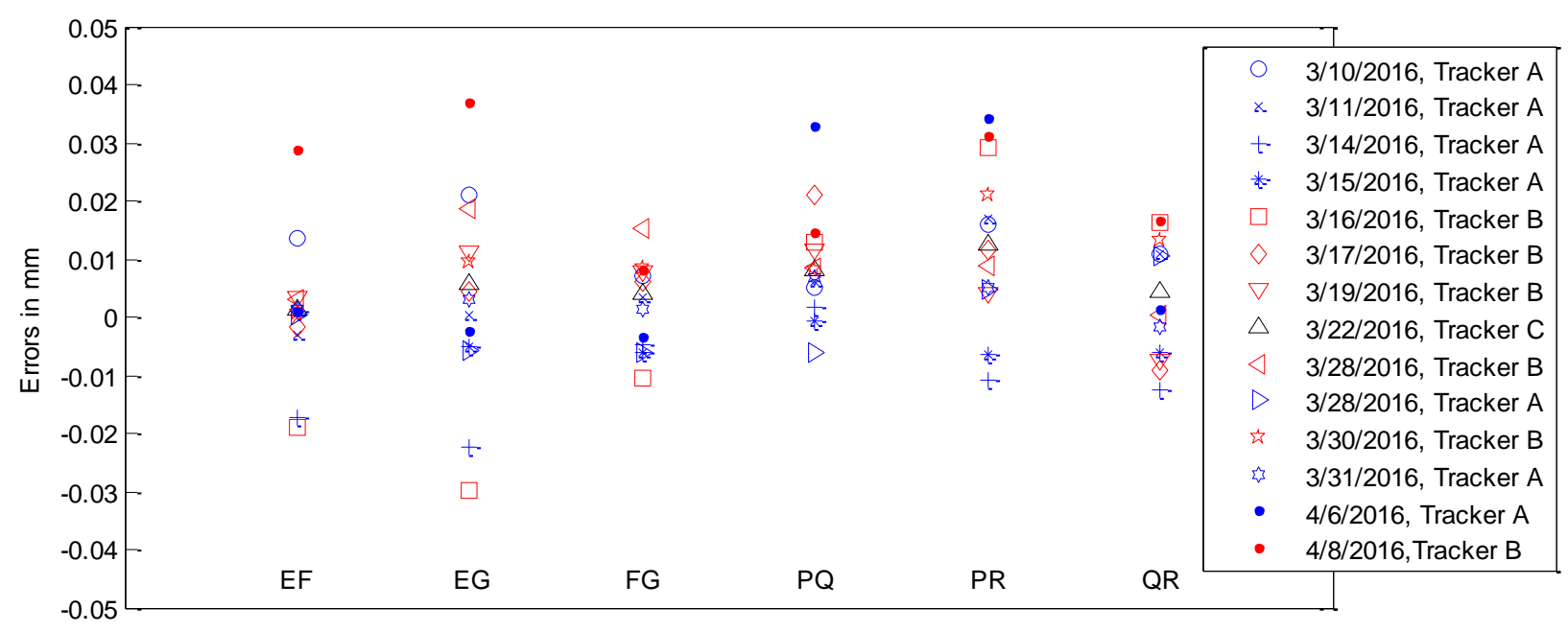

Fig. 8 Errors in three horizontal and three diagonal lengths over a one-month period

\subsubsection{Uncertainty budget}

Mechanical terms: The uncertainty in locating a single point in space resulting from error sources associated with the laser tracker, the centering of the integration sphere with the SMR, and the concentricity of optical and mechanical center of the SMR is first determined. The point coordinate uncertainty is then propagated to estimate the uncertainty in the point-to-point length.

Tracker contribution: Because of the averaging from front-face and back-face, and averaging achieved as a result of measurements from four positions that samples different regions of the azimuth and elevation angle encoders, mostly random error components remain. Therefore, the experimentally determined one standard deviation repeatability of $0.1 \mathrm{mdeg}$ is taken as the standard uncertainty in the angles measured by the tracker. At a distance of $6 \mathrm{~m}$ (which is the farthest distance the tracker is from the grid), the standard uncertainty along the horizontal and vertical angle axes are $11 \mu \mathrm{m}$ each. At a distance of $6 \mathrm{~m}$, the tracker is sufficiently far enough from the grid that the component of the tracker's ranging error along the direction of the length is negligible, therefore only the angular errors of the tracker are considered.

Integration sphere concentricity with SMR: The concentricity of all 14 integration spheres are measured on a CMM. The experimentally measured one standard deviation is $5 \mu \mathrm{m}$ and this value is considered as the standard uncertainty in the determination of the coordinate.

SMR concentricity: The concentricity specification of the physical and optical center of all SMRs used is $2.5 \mu \mathrm{m}$. Assuming this value as the upper bound with any value within that bound as equally probable, the one standard deviation uncertainty in the measured coordinate is $1.4 \mu \mathrm{m}$. 
Standard uncertainty in the length: The terms above result in a combined standard uncertainty of $12 \mu \mathrm{m}$ for a point coordinate or $12 \sqrt{2}=17 \mu \mathrm{m}$ for a point-to-point length.

Thermal effects: The diagonals are the longest point-to-point lengths in the grid and they span $3.2 \mathrm{~m}$ horizontally on the concrete floor and $3.2 \mathrm{~m}$ vertically on aluminum rails. Assuming $\pm 0.5{ }^{\circ} \mathrm{C}$ fluctuation of the room temperature as the upper bound with any value within that bound as equally probable, and assuming expansion coefficients of $22 \times 10^{-6} /{ }^{\circ} \mathrm{C}$ for aluminum and $12 \times 10^{-6} /{ }^{\circ} \mathrm{C}$ for concrete, the standard uncertainty in the length is determined to be $22 \mu \mathrm{m}$. The uncertainty due to changes in refractive index (and therefore measured range) is not considered because that is an order of magnitude smaller than the uncertainty due to thermal expansion of the floor/rail.

Combined standard uncertainty: Combining the mechanical contribution of $17 \mu \mathrm{m}$ and the thermal contribution of $22 \mu \mathrm{m}$, the combined standard uncertainty in the length is determined to be $28 \mu \mathrm{m}$. The expanded uncertainty $(k=2)$ is $56 \mu \mathrm{m}$.

\section{The inside test}

\subsection{Test setup}

The inside test consists of two integration spheres placed about $6 \mathrm{~m}$ apart from each other, and facing each other (see Fig. 9). The 3D imaging system is placed inline, in between, and equidistant from the two spheres. The center-to-center distance between the spheres is calibrated using a laser tracker and three registration nests.

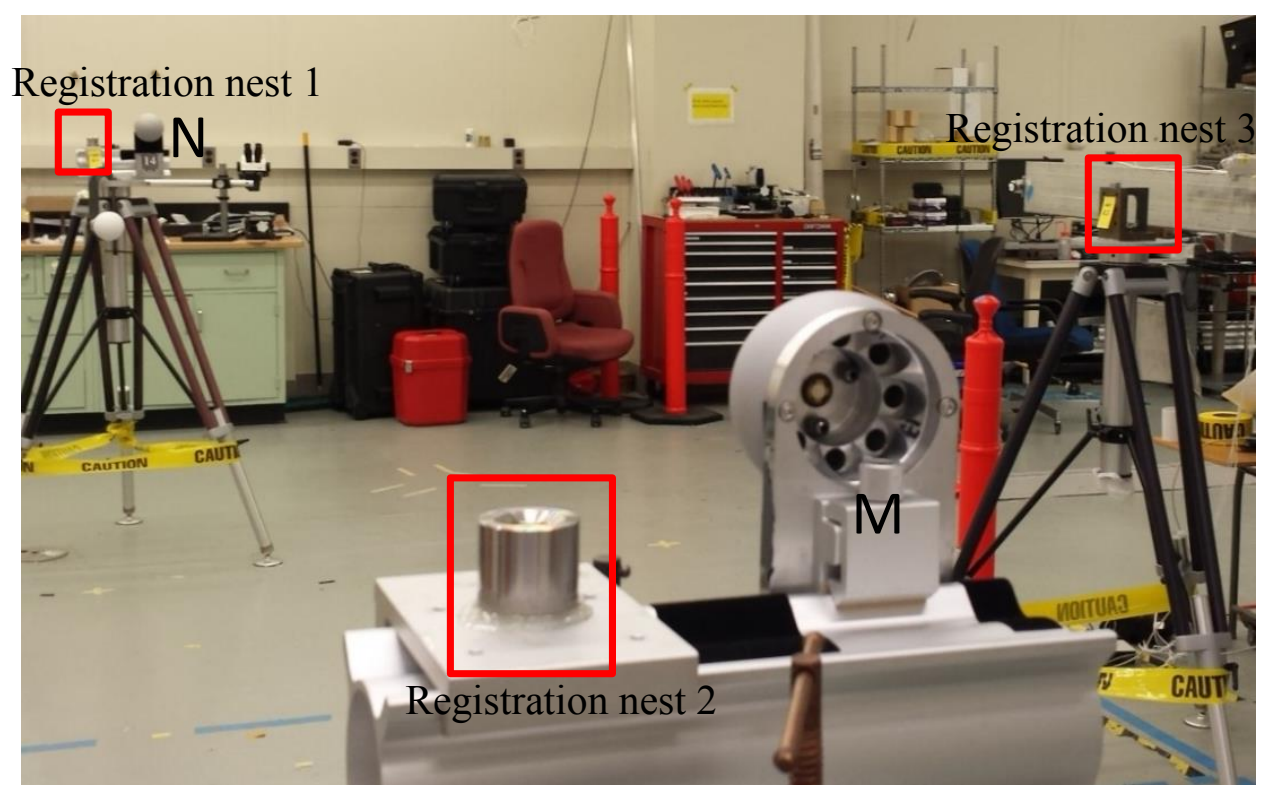

Fig. 9 The inside test setup showing integration spheres $M$ and $N$, and the three registration nests 


\subsection{Calibration procedure}

The inside length is calibrated using a laser tracker in conjunction with three registration nests. Two of the registration nests are placed next to the integration spheres, while the third is placed at approximately the mid-point but slightly offset from the line joining the two ends of the length (see Fig. 10). The tracker is placed on one side of the setup (Position 1) and the coordinate of the center of the integration sphere closest to the tracker (integration sphere 1) is determined in a coordinate system established using the registration nests. The tracker is then moved to the other side (Position 2), and the coordinate of the center of the other integration sphere (integration sphere 2) is determined in the same coordinate system established again using the registration nests. Because the integration sphere centers are measured in the same coordinate system, the integration sphere center-to-center distance is simply the Euclidean distance between the two measured sphere center coordinates.

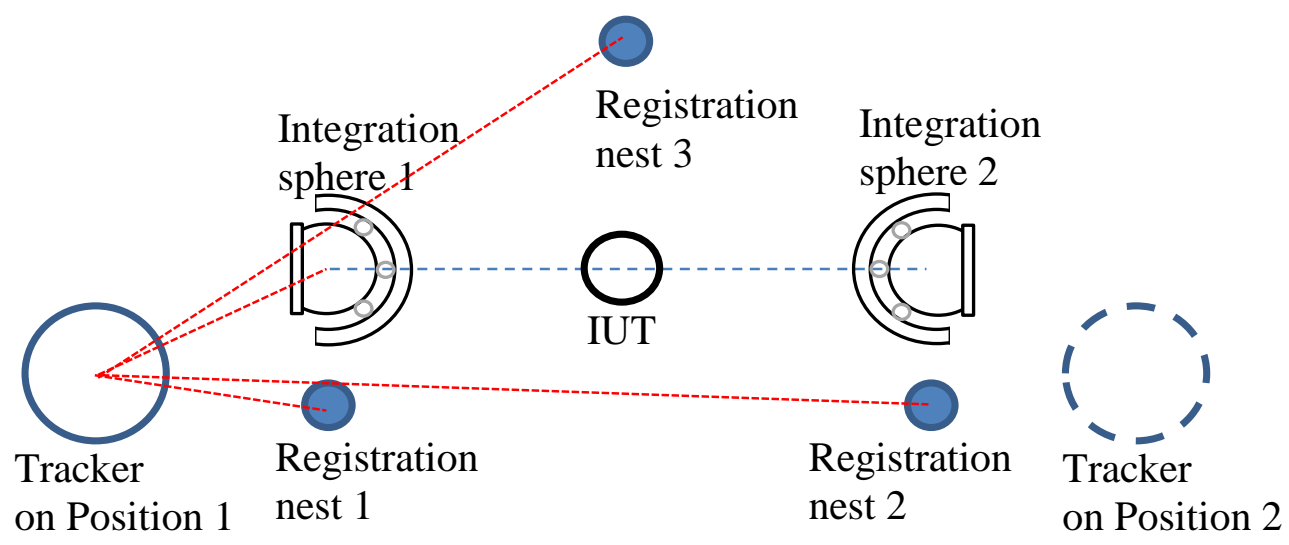

Fig. 10 Calibrating the center-to-center distance

As a point of detail, the tracker measurements in the inside calibration are always performed by averaging front-face and back-face measurements. Further, in order to reduce the influence of systematic errors, tracker measurements are performed from four different azimuth orientations (each $90^{\circ}$ apart) from each position. That is, with the tracker placed on Position 1 (see Fig. 10), the three registration nests and the center of integration sphere 1 are first measured (all of them as the average of front and back-face). A coordinate system is established with registration nest 1 as the origin, nest 2 defines the $X$ axis and nest 3 the $\mathrm{XY}$ plane. The coordinates of the center of the integration sphere in that coordinate system is determined. The tracker then is rotated by approximately $90^{\circ}$, the three nests and the center of the integration sphere are measured, and the coordinates of the integration sphere center is again determined. This process is repeated another two times, and all four sphere center coordinates are averaged. A similar process is performed with the tracker on Position 2 . 


\subsection{Validation experiments}

In order to determine the magnitude of the errors associated with registration, a simple experiment is performed. The integration spheres are replaced with $38.1 \mathrm{~mm}$ (1.5 in) SMR nests, referred to as test nests in Fig. 11. The center-to-center distance between the two test nests is calibrated using a tracker placed along the line joining these two nests. The registration based length between the same nests is then determined according to the procedure described Section 4.2. Table 2 shows the difference between the registrationbased length and the line-of-sight calibration performed on four different days. The errors are all smaller than $10 \mu \mathrm{m}$, well within the uncertainty claimed in the next section.

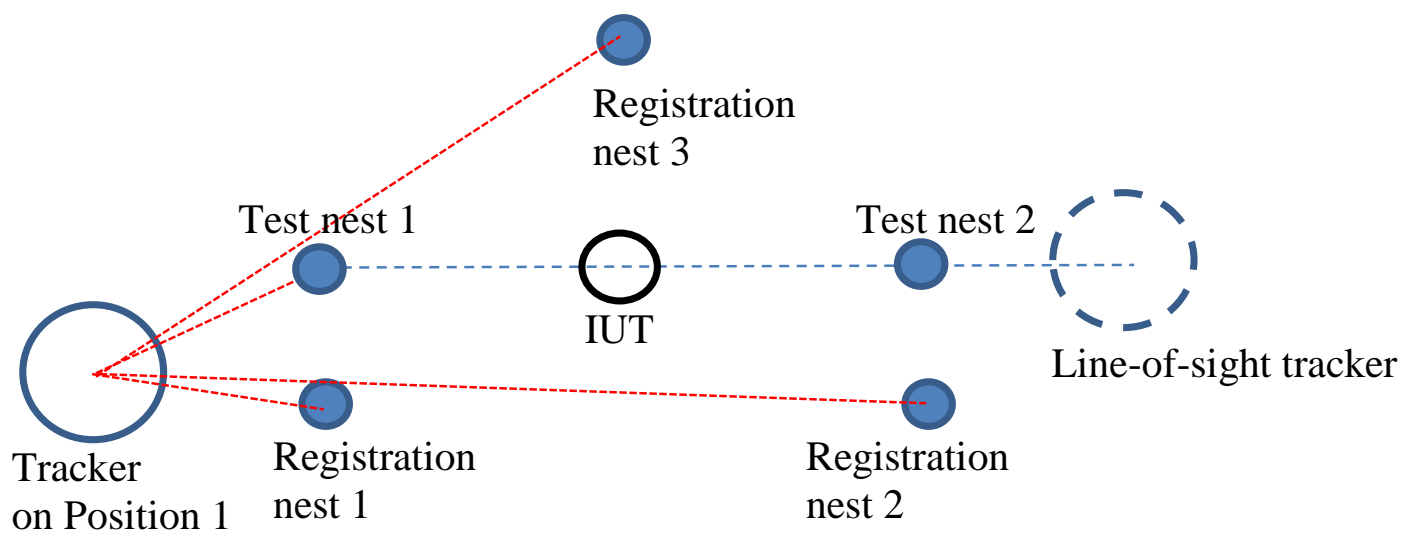

Fig. 11 Schematic of a setup to validate the registration based center-to-center measurement.

Table 3 Registration based length compared against line-of-sight calibration

\begin{tabular}{|l|l|l|l|}
\hline & $\begin{array}{l}\text { Line-of- } \\
\text { sight } \\
\text { reference } \\
(\mathrm{mm})\end{array}$ & $\begin{array}{l}\text { Registration } \\
\text { based } \\
\text { measurement } \\
(\mathrm{mm})\end{array}$ & Error (mm) \\
\hline Set 1 & 6414.578 & 6414.572 & -0.006 \\
\hline Set 2 & 6414.582 & 6414.578 & -0.004 \\
\hline Set 3 & 6414.582 & 6414.585 & 0.003 \\
\hline Set 4 & 6414.580 & 6414.585 & 0.005 \\
\hline
\end{tabular}

\subsection{Uncertainty budget}

Mechanical terms: The uncertainty in locating a single point in space resulting from error sources associated with the laser tracker, the centering of the integration sphere with the SMR, and the concentricity of optical and mechanical center of the SMR is first determined. The point coordinate uncertainty is then propagated to estimate the uncertainty in the point-to-point length.

Tracker contribution: Monte Carlo Simulation (MCS) of the registration based measurement process indicates a standard uncertainty of $8 \mu \mathrm{m}$ in measuring the 
coordinate of each of the targets. As in the case of the grid calibration, the manufacturer specifications are not used as input to the MCS. Instead, the experimentally determined one standard deviation repeatability of $0.1 \mathrm{mdeg}$ is considered as the standard uncertainty in the angles measured by the tracker, and this value is scaled by the measured range to the targets. This translates to a standard uncertainty of about $2 \mu \mathrm{m}$ at the near target (which is nominally $1 \mathrm{~m}$ away from the tracker) and $12 \mu \mathrm{m}$ at the far target (nominally $7 \mathrm{~m}$ away) along the transverse directions. Along the ranging direction, the manufacturer's specification of $10 \mu \mathrm{m}$ is used as the upper bound and any value inside of that is considered as equally probable. It is noted the tracker used here is from a different manufacturer than the one used in the experiments described in Section 3.2.3, hence the different MPE.

Integration sphere concentricity with SMR: The concentricity of all 14 integration spheres are measured on a CMM. The experimentally measured one standard deviation is $5 \mu \mathrm{m}$ and this value is considered as the standard uncertainty in the determination of the coordinate.

SMR concentricity: The concentricity specification of the physical and optical center of the SMRs is $2.5 \mu \mathrm{m}$. Assuming this value as the upper bound with any value within that bound as equally probable, the one standard deviation uncertainty in the measured coordinate is $1.4 \mu \mathrm{m}$.

Standard uncertainty in the length: The terms above result in a combined standard uncertainty of $10 \mu \mathrm{m}$ for a point coordinate or $10 \sqrt{2}=14 \mu \mathrm{m}$ for a point-to-point length.

Thermal effects: Assuming $\pm 0.5^{\circ}$ fluctuation in temperature in the room as upper bound with any value inside that bound as equally probable, and assuming thermal expansion coefficient of $12 \times 10^{-6} /{ }^{\circ} \mathrm{C}$ for concrete, the standard uncertainty is determined to be $21 \mu \mathrm{m}$ for a $6 \mathrm{~m}$ length. As in the case of the grid calibration, the uncertainty due to changes in refractive index (and therefore measured range) is not considered because that is an order of magnitude smaller than the uncertainty due to thermal expansion of the floor.

Combined standard uncertainty: Combining the mechanical contribution $14 \mu \mathrm{m}$ and the thermal contribution of $21 \mu \mathrm{m}$, the combined standard uncertainty in length is determined to be $25 \mu \mathrm{m}$. The expanded uncertainty $(k=2)$ is $50 \mu \mathrm{m}$.

\section{Relative range tests}

\subsection{Test setup}

The relative range tests are performed in the $60 \mathrm{~m}$ tape-tunnel facility at NIST (see Fig. 12). The $3 \mathrm{D}$ imaging system is placed on one end of the tape tunnel while the laser tracker is placed at the other end. Relative range tests are first performed with the plate-sphere artifact and subsequently performed again with the bare-sphere artifact. The near position of the artifact from the 3D imaging system is generally specified by the manufacturer while 
a few test scans are performed to determine the far position. For most of the systems tested during the runoff, the near position was between $1 \mathrm{~m}$ to $3 \mathrm{~m}$ while the far position was the full length of tunnel, i.e., $60 \mathrm{~m}$.
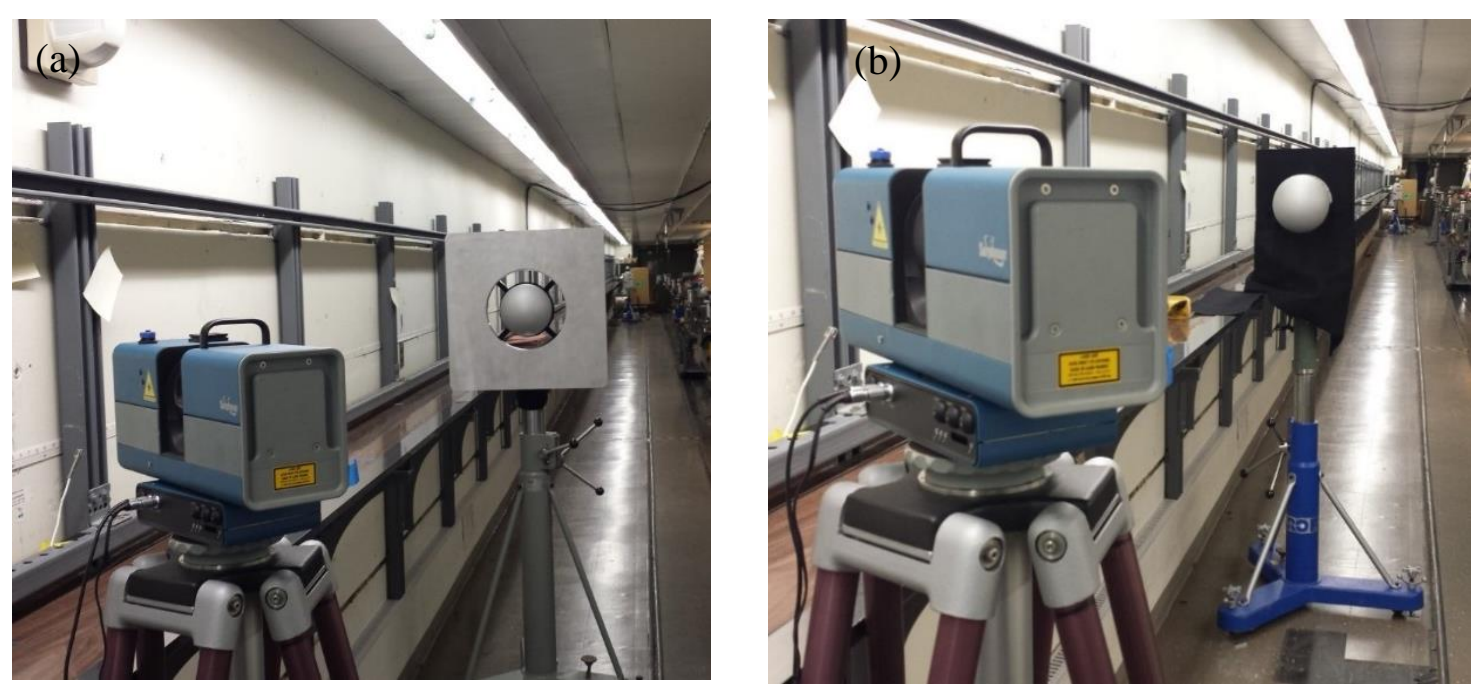

Fig. 12 Relative range tests showing a $3 D$ imaging system placed in front of a) the platesphere artifact and $b$ ) the bare-sphere artifact. The laser tracker is at the far end of the tape-tunnel.

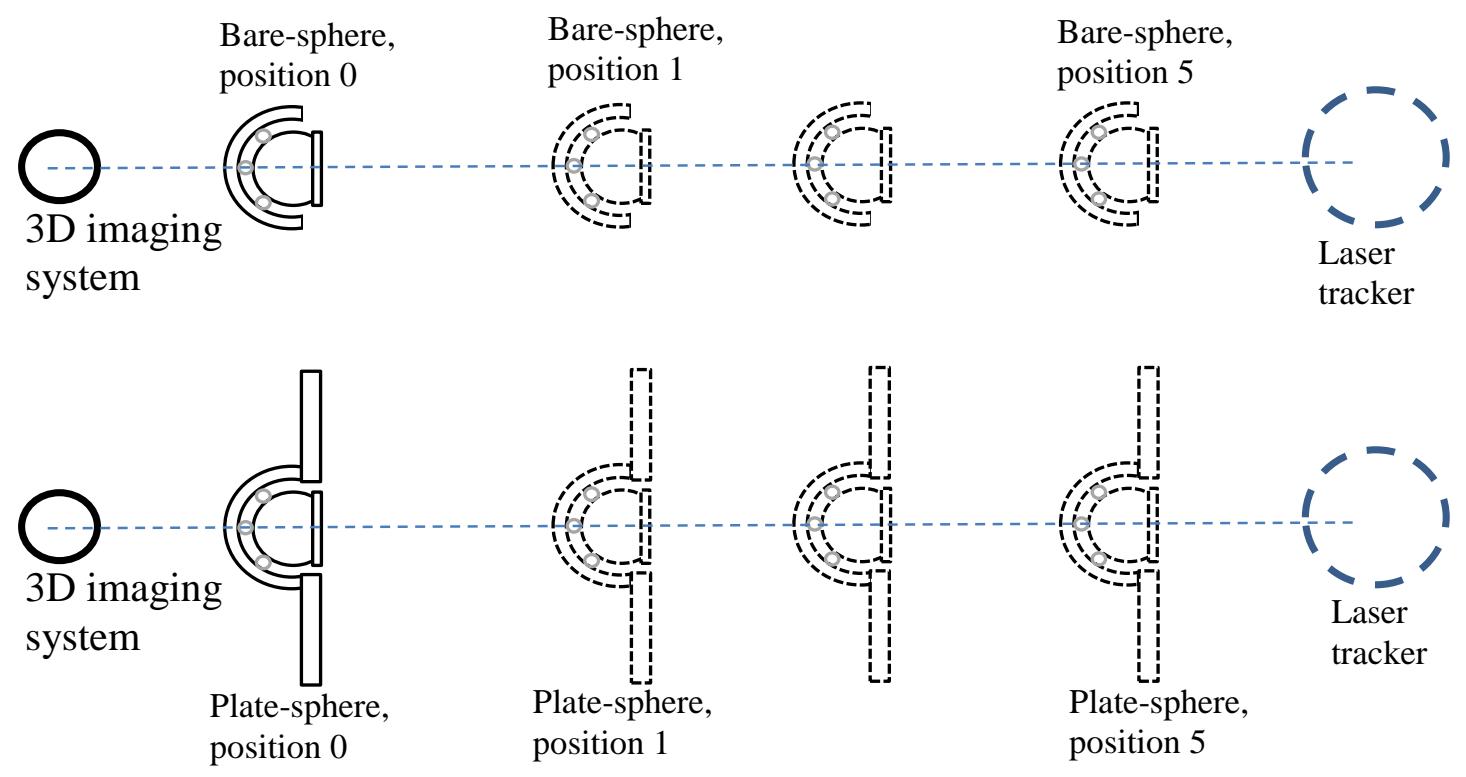

Fig. 13 Relative range tests using the bare-sphere and the plate-sphere artifact

The artifact is first placed at the near position (position 0 in Fig. 13), facing the 3D imaging system, and approximately centered on the line joining the 3D imaging system and the laser tracker. This position is the reference for all displacement calculations. Data is 
simultaneously acquired from both the instruments. The artifact is then moved to positions $1,2,3,4$, and 5 while data are acquired from both instruments at each position. Displacement as recorded by both instruments (with respect to position 0 ) and the relative range error are calculated.

Fig. 12 shows a 3D imaging system in the foreground, the artifact placed in front of it, and a laser tracker at the far end of the tape-tunnel. Fig 13 shows a schematic of the relative range test using the bare-sphere and the plate-sphere artifact.

\subsection{Uncertainty budget}

The relative range test involves measuring the displacement between two positions of the target, a reference position (denoted by position 0 in Fig. 13) and a test position (any of positions 1 through 5 in Fig. 13), both of which are along a radial direction of the 3D imaging system. The reference position of the target (position 0) is assumed to be $61 \mathrm{~m}$ from the laser tracker for the uncertainty calculations. The test position (positions 1 through 5 ) of the target may be anywhere between the laser tracker and position 0 of the target.

Primary contributors to the uncertainty in the displacement measurement are the errors associated with the range measurement of the laser tracker and the thermal environment. The thermal expansion of the concrete floor is not of any consequence in this case because both instruments measure the range to the artifact simultaneously.

A general equation for the uncertainty in determination of the range to any of the target positions is first presented. The uncertainty in the displacement is then calculated for some example lengths.

Tracker contribution: Using manufacturer specification of $10 \mu \mathrm{m}$ as upper bound and assuming rectangular distribution, the one standard deviation point coordinate uncertainty is determined to be $5.8 \mu \mathrm{m}$ along the ranging direction.

Temperature contribution: The temperature sensor of the laser tracker only monitors the temperature at one point along the laser path. Because long lengths are measured, the tracker is unable to adequately compensate for the changing thermal environment. Therefore, the uncertainty in range measurements because of temperature fluctuations along the laser path is considered. Assuming $\pm 1{ }^{\circ} \mathrm{C}$ fluctuation in temperature along the laser path with any value inside that bound as equally probable, and with temperature influence of $1 \times 10^{-6} /{ }^{\circ} \mathrm{C}$ (this is due to the change in refractive index based on Edlén's equation), the standard uncertainty in range measurement at a distance of $r$ meters is $r / \sqrt{3}$ in units of micrometers.

The uncertainty in determining range to the target is the root sum square of the two terms, thus it is $\sqrt{\frac{r^{2}}{3}+36}$ in units of micrometers. The uncertainty in the displacement is the root sum square of the uncertainty in the range at two ends of the length. Table 4 presents the uncertainty for some displacement measurements. 
The expanded $(\mathrm{k}=2)$ uncertainty for the ranging measurements varies between $92 \mu \mathrm{m}$ for a $12 \mathrm{~m}$ length to $72 \mu \mathrm{m}$ for a $60 \mathrm{~m}$ length. One of the drawbacks of positioning the laser tracker at the opposite end of the tape tunnel from the 3D imaging system is obvious from Table 4. The uncertainty in the reference measurement is larger for smaller displacements. This is because a small displacement of the target with respect to the $3 \mathrm{D}$ imaging system requires measuring two positions that are both far away from the laser tracker, hence the larger uncertainty.

Table 4 Uncertainty for some displacement measurements

\begin{tabular}{|l|l|l|l|l|l|l|}
\hline $\begin{array}{l}\text { Test } \\
\#\end{array}$ & $\begin{array}{l}\text { Range to } \\
\text { the } \\
\text { reference } \\
\text { position }(\mathrm{m})\end{array}$ & $\begin{array}{l}\text { Uncertainty in } \\
\text { the reference } \\
\text { position }(\mu \mathrm{m})\end{array}$ & $\begin{array}{l}\text { Range to } \\
\text { the test } \\
\text { position } \\
(\mathrm{m})\end{array}$ & $\begin{array}{l}\text { Uncertainty } \\
\text { in the test } \\
\text { position }\end{array}$ & $\begin{array}{l}\text { Displac- } \\
\text { ement } \\
(\mathrm{m})\end{array}$ & $\begin{array}{l}\text { Standard } \\
\text { uncertainty in } \\
\text { the } \\
\text { displacement } \\
(\mu \mathrm{m})\end{array}$ \\
\hline 1 & 61 & 36 & 49 & 29 & 12 & 46 \\
\hline 2 & 61 & 36 & 37 & 22 & 24 & 42 \\
\hline 3 & 61 & 36 & 25 & 16 & 36 & 39 \\
\hline 4 & 61 & 36 & 13 & 10 & 48 & 37 \\
\hline 5 & 61 & 36 & 1 & 6 & 60 & 36 \\
\hline
\end{tabular}

It should be noted that the temperature variation in the tape tunnel is generally within $\pm 0.2^{\circ}$ and therefore the uncertainty in the realization of a $60 \mathrm{~m}$ length is about $1 / 5^{\text {th }}$ the amount claimed in Table. 4. The reason for the conservative estimate of $\pm 1{ }^{\circ} \mathrm{C}$ fluctuation in temperature is because of the large number of people in the room during the runoff.

\section{Summary}

This report describes the facilities established by the DMG at NIST to realize all of the test procedures in the current draft of the ASTM E57.02 standard for point-to-point performance evaluation of spherical coordinate 3D imaging systems. This report describes the test setups, calibration procedures, validation data, and uncertainty budgets.

\section{Acknowledgements}

The authors thank all participants of the instrument runoff and the biweekly E57 meetings for supporting this standards effort, Dr. Kam Saidi and Dr. Steve Phillips for helpful discussions, and Dr. Craig Shakarji and Dr. Marek Franaszek for carefully reviewing this document.

\section{References}

1. WK43218. ASTM E57.02, New test methods for evaluating the performance of medium-range, spherical coordinate, 3D imaging systems for point-to-point distance measurements 
2. NISTIR 8152, Report on the May 2016 ASTM E57.02 instrument runoff at NIST, Part 1 - Background information and key findings, 2016

3. B. Muralikrishnan, S. Phillips, D. Sawyer, Laser trackers for large scale dimensional metrology - A review, Precision Engineering, 44, 2016, p. 13-28

4. B. Muralikrishnan, C. Blackburn, D. Sawyer, S. Phillips, and R. Bridges, Measuring scale errors in a laser tracker's horizontal angle encoder through simple length measurement and two-face system tests, Journal of Research of the NIST, 115 (5), p.291-301, 2010 